\title{
Optimal Reinsurance Design for Pareto Optimum: From the Perspective of Multiple Reinsurers
}

\author{
Xing Rong ${ }^{1}$ and Yunzhou $\mathrm{Zhu}^{2}$ \\ ${ }^{1}$ Southwestern University of Finance and Economics, Chengdu, China \\ ${ }^{2}$ Department of Actuary, China Continent Property \& Casualty Insurance Company Ltd., Shanghai, China \\ Correspondence should be addressed to Yunzhou Zhu; zyzzju1988@126.com
}

Received 13 May 2016; Revised 25 August 2016; Accepted 30 August 2016

Academic Editor: Laura Gardini

Copyright (c) $2016 \mathrm{X}$. Rong and Y. Zhu. This is an open access article distributed under the Creative Commons Attribution License, which permits unrestricted use, distribution, and reproduction in any medium, provided the original work is properly cited.

\begin{abstract}
This paper investigates optimal reinsurance strategies for an insurer which cedes the insured risk to multiple reinsurers. Assume that the insurer and every reinsurer apply the coherent risk measures. Then, we find out the necessary and sufficient conditions for the reinsurance market to achieve Pareto optimum; that is, every ceded-loss function and the retention function are in the form of "multiple layers reinsurance."
\end{abstract}

\section{Introduction}

The insurance and reinsurance industry has an irreplaceable role in global economy risk compensation. With global warming and the earth being ever-active, various types of extreme disasters are happening more frequently; therefore, research on loss allocation by reinsurance is of more practical significance. In October 2012, hurricane Sandy landed in the United States and more than 650,000 houses were destroyed, resulting in economic losses of up to $\$ 70$ billion. Insurance paid 18.75 billion US dollars, of which $60 \%$ were paid by primary insurers while the other $40 \%$ were by reinsurance ones. In 2001, the 911 terrorist attacks suffered by the United States caused huge losses and insurance indemnity reached 26.8 billion US dollars. Of these, 35\% were paid by primary insurance companies and $65 \%$ by reinsurance ones (the data comes from Global Reinsurance forum). The examples above show the ceded-loss strategy of reinsurance companies plays a crucial role in reducing losses encountered in catastrophes. The superiority of reinsurance mechanism is mainly reflected in the following two aspects. First, the reinsurance can reduce the exposure risk and thus stabilize underwriting income. Catastrophe risk is characteristic of low occurrence and huge loss. Consequently, a single claim may lead to financial crisis of the entire insurance company or even bankruptcy. Therefore, the reinsurance strategy allows insurance companies to avoid or reduce the impact of catastrophe risks. Undoubtedly, for some newly established insurance companies, the reinsurance can significantly improve their risk tolerance abilities. Second, by undertaking reinsurance, the primary insurance companies not only increase income the primary insurance companies not only increase income.

With the development of economy, the value of the subject matter insured has been increasing. The solvency faced by separate original insurers is facing challenges; thus, seeking multiple insurers in international reinsurance market to share the risk and revenue is becoming popular. The total worldwide premium income from reinsurance has risen from $20 \%$ in the 1980 s of last century to $40 \%$ today, showing that the value of reinsurance has improved significantly. Appropriately designed reinsurance programs make it possible to fully realize the advantages of the reinsurance mechanism. However, an improper reinsurance program probably leads to high risk aggregation and loss of quality services, and it will further affect underwriting profits and even endanger the solvency. Thus, the studying on the optimal reinsurance design has practical significance. 
For individuals and the whole society, risks exist objectively. The final risk takers often include policy holders, insurers, reinsurers, financial market investors, and also local and central government. Among them, the policy holders, insurers, reinsurers, and financial market investors are marketbased sharers, whereas local and central governments provide non-market-based sharing. Non-market-based sharing may lead to distortions of the market; therefore, market-based sharing is relatively more efficient than it. Currently, the insurance risk sharing through capital market is still in its infant stage. The core of market-based loss sharing lies in sharing by both the insurers and the international reinsurance markets. Therefore, from the perspective of theoretical research, optimal reinsurance design has always been one of the most fascinating problems in the actuarial research fields.

Great attention has been paid from both academics and practitioners to the study of optimal reinsurance problems since the seminal work of Borch [1]. In the past half century, a variety of optimal reinsurance designs have been devised by either minimizing certain risk measure of an insurer's risk exposure or maximizing the expected utility of the final wealth of a risk-averse insurer; see, for example, Borch [1], Arrow [2], Raviv [3], Huberman et al. [4], Young [5], Kaluszka [6], Kaluszka and Okolewski [7], and references therein. Recently, the Value-at-Risk (VaR) and Conditional Value atRisk (CVaR) based optimal reinsurance designs have been extensively studied due to the prevalent use of these two risk measures in financial and insurance practice; see Cai and Tan [8], Cai et al. [9], Balbás et al. [10], Tan et al. [11, 12], Cheung [13], and Chi and Tan $[14,15]$, just to name a few.

The proposed problem can be classified as a multiobjective optimization problem. This paper just uses the idea of multiobjective optimization but does not involve the algorithm. In 1896, the earliest idea of multiobjective optimization originates from the French economist Pareto, who proposed it in the study of economic equilibrium. Subsequently, this problem has gradually developed in three different areas, namely, operations research, economics, and psychology. Currently, multiobjective programming has been applied to fields like engineering [16], portfolio construction [17], risk management of interest rate [18], agricultural management [19], and accounting [20]. Zopounidis et al. [21] came up with the statistics that, during the period of 2002-2014, a total of 644 research papers had been devoted to application of multiple-criteria decision analysis to the financial field.

All the optimal reinsurance models in the aforementioned literature are formulated under the assumption that the insurer cedes risks to a single reinsurer. In practice, with the development of the insurance industry, the insurance coverage is also expanding. Therefore, the insurer prefers finding multiple reinsurers to share the risks and the underwriting revenue. Nowadays, the scale of reinsurance is becoming larger and larger; thus, a perfect market of reinsurance has been created. There exist various types of reinsurance contracts in such a market; most of them are in the case that the insurer cedes the risk to several reinsurers. Thus, we will focus on the problem of "ceding the risk to multiple reinsurers."
In this paper, we apply the principle in microeconomics that "Pareto optimum achieves the optimal Nash equilibrium" as a starting point and then analyze the situation in which the original insurers allocate risks to several reinsurers. Assume that the insurer and all reinsurers use coherent distortion risk measures; we demonstrate the necessary and sufficient conditions for the reinsurance market to achieve Pareto optimum. That is, every reinsurance function should be "multiple-layer reinsurance." The novelty and contribution of this paper are summarized in the following aspects.

First, we study the optimal reinsurance on a global perspective. Different from the literatures mentioned above, we focus on the optimization problem of the insurer with multireinsurers. This assumption is closer to the insurance practice since the insurer's optimal reinsurance contract does not always mean the best choice for the reinsurer. Furthermore, if the insurer only seeks a single reinsurer in the reinsurance contracts, that may lead to serious problems such as inadequate solvency and credit risks. Thus, the primary insurers often seek multiple reinsurers to share the risks.

Second, our reinsurance model design does not have any assumptions on the premium principles and is thus broadly applicable. In previous literatures, the optimal designs of multireinsurers have all been based on certain specific premium principle assumption. Many of them indicate that the future research is needed for their optimum reinsurance designs to be applicable under other premium principles. This paper has addressed such a problem. By reducing the assumptions on the premium principles, our research makes it more generally applicable.

Third, the reinsurance model has outstanding economic significance. To achieve the optimal reinsurance design with multireinsurers, the concept "Pareto optimum" from microeconomics is adopted in this paper. This makes our proposed reinsurance model different from those in existing actuarial literatures and possess more economic value.

This paper proceeds as follows. Our optimal reinsurance model is formulated in Section 2, and its optimal solutions are constructed and analyzed in Section 3. Finally, two numerical samples and a brief prospection are carried out at Section 4.

\section{Model Formulation}

Consider an insurer suffers a potential loss $X$, where $X$ is a nonnegative random variable defined on a probability space $(\Omega, \mathscr{G}, \mathbb{P})$ with cumulative distribution function (CDF).

$F_{X}(x) \triangleq \mathbb{P}(X \leq x)$ and $0<\mathbb{E}[X]<\infty$. In order to reduce its risk exposure, the insurer cedes part of loss to $\mathrm{m}$ reinsurers. Mathematically, we let $f_{j}(X)$ represent the loss ceded to the $j$ th reinsurer, $j=1, \ldots, m$. Consequently, an $m$-dimensional vector $\mathbf{f}=\left(f_{1}, \ldots, f_{m}\right)$ can be defined as the ceded risk suffered by $m$ reinsurers. Moreover, the retention of an insurer is $R_{\mathbf{f}}(X)=X-\sum_{j=1}^{m} f_{j}(X) . f_{j}(\cdot)$ and $R_{\mathbf{f}}(\cdot)$ are thus called the ceded-loss function and the retained loss function, respectively. To mitigate the potential moral hazard, we follow the convention and assumption that the insurer and all reinsurers are subject to a larger claim payment upon a larger realized loss on the insurance policies 
issued to the insured. Mathematically, it is equivalent to the assumption that both the ceded-loss functions and the retained loss function are increasing. It leads to the following feasible set of ceded-loss functions:

$$
\begin{aligned}
\mathscr{G}_{m} & =\left\{\mathbf{f} \mid \mathbb{R}_{m}^{+} \longrightarrow \mathbb{R}_{m}^{+}: 0 \leq f_{j}(x)\right. \\
& \leq x, R_{\mathbf{f}} \text { and every } f_{j}, j \\
& =1, \ldots, m \text { are increasing functions }\} .
\end{aligned}
$$

Obviously, $R_{\mathbf{f}}$ and each $f_{j}$ are Lipschitz continuous since we observe

$$
\begin{array}{r}
0 \leq f_{j}\left(x_{2}\right)-f_{j}\left(x_{1}\right) \leq h_{m}\left(x_{2}\right)-h_{m}\left(x_{1}\right) \leq x_{2}-x_{1}, \\
\forall 0 \leq x_{1} \leq x_{2}, j=1, \ldots, m,
\end{array}
$$

where $h_{m} \triangleq \sum_{j=1}^{m} f_{j}$.

While the insurer can reduce its risk exposure by ceding part of the loss, it incurs an additional cost in the name of reinsurance premium. Assume that the reinsurance premium for the $j$ th reinsurer is calculated according to a premium principle $\Pi_{j}(\cdot)$, which is a functional mapping from nonnegative random variables $X$ to the set of nonnegative real numbers with $\Pi_{j}(0)=0$. As we know, there are many premium principles used in insurance practice, for example, expectation principle and Wang's principle. However, it will be observed that any assumption on premium is meaningless in our model. Furthermore, the risk measure is the unique factor to determine the optimal reinsurance functions $f_{j}$. Although VaR and CVaR are popular in reinsurance analysis, both of them are the special cases of the distortion risk measure which is firstly introduced by Wang [22]. In order to rightly analyze the optimal reinsurance, the mathematical definition of the distortion risk measure is presented as follows.

Definition 1. Assume that there exists an increasing function $G:[0,1] \rightarrow[0,1]$ which satisfies $G(0)=0$ and $G(1)=1$; then, the distortion risk measure of random variable $X$ is defined as

$$
\rho_{G}(X) \triangleq \int_{0}^{+\infty} G\left(S_{X}(t)\right) \mathrm{d} t
$$

where $S_{X}(t) \triangleq 1-F_{X}(t)$ and $G(\cdot)$ can be called the generation function.

Now let us present the concept of "Pareto optimum" before the reinsurance model is established. Popularly, assume that there exist a group of people and resources which can be allocated. Changing from one dispensing state to another, no one can be made better off by making someone worse off. Such a state or distribution of economic resources is called the Pareto optimum. Later researchers have conducted a series of studies on such a state; the most famous one is American economist John. F. Nash. Combining game theories, he proved that Pareto optimum is a specific form of "Nash equilibrium" which means the economic activities are the most efficient and ideal.
Definition 2. Given a random return variable $Y, Y \geq 0$, (a.s.), we divide it into $n$ parts such that $\sum_{j=1}^{n} Z_{j}=Y$, where $\mathbf{Z}=\left(Z_{1}, \ldots, Z_{n}\right)$. Additionally, we consider the following optimization problem:

$$
\max _{\mathbf{Z} \in D_{n}(Y)}\left\{\mathbb{E}\left[U_{1}\left(Z_{1}\right)\right], \ldots, \mathbb{E}\left[U_{n}\left(Z_{n}\right)\right]\right\},
$$

where $U_{j}$ is the utility function and $D_{n}(Y)=\left\{\mathbf{Z} \mid \sum_{j=1}^{n} Z_{j}=\right.$ $Y\}$. Any fixed $\mathbf{Z} \in D(Y)$ is called a Pareto division. For a group of Pareto divisions $\mathbf{Z}^{*}=\left(Z_{1}^{*}, \ldots, Z_{n}^{*}\right)$, if there does not exist another group of divisions $\mathbf{Z}^{\prime}=\left(Z_{1}^{\prime}, \ldots, Z_{n}^{\prime}\right)$ such that every inequality $\mathbb{E}\left[U_{j}\left(Z_{j}^{\prime}\right)\right] \geq \mathbb{E}\left[U_{j}\left(Z_{j}^{*}\right)\right], j=1, \ldots, n$, holds and at least one inequality $\mathbb{E}\left[U_{j}\left(Z_{j}^{\prime}\right)\right]>\mathbb{E}\left[U_{j}\left(Z_{j}^{*}\right)\right]$ holds, then we say $\mathbf{Z}^{*}$ achieves Pareto optimum.

In reinsurance market, all insurers and reinsurers pay more attention to the risks. As an alternative, we rewrite Definition 2. Suppose that $Y$ is a random variable; then, problem (4) can be rewritten as

$$
\min _{\mathbf{Z} \in D_{n}(Y)}\left\{\rho_{1}\left(Z_{1}\right), \ldots, \rho_{n}\left(Z_{n}\right)\right\}
$$

where $\rho_{j}, j=1, \ldots, n$ are given risk measures. For a group of Pareto divisions $\mathbf{Z}^{*}=\left(Z_{1}^{*}, \ldots, Z_{n}^{*}\right)$, if there is no division $\mathbf{Z}^{\prime}=\left(Z_{1}^{\prime}, \ldots, Z_{n}^{\prime}\right)$ such that every inequality $\rho_{j}\left(Z_{j}^{\prime}\right) \leq \rho_{j}\left(Z_{j}^{*}\right)$, $j=1, \ldots, n$, holds and at least one inequality $\rho_{j}\left(Z_{j}^{\prime}\right)<\rho_{j}\left(Z_{j}^{*}\right)$ holds, then we say $\mathbf{Z}^{*}$ achieves the Pareto optimum. However, due to the various different risk measures, problem (5) is hard to be solved. Fortunately, Jouini et al. [23] and Burgert and Ruschendorf [24] have proved the following: if $\rho_{j}(\cdot), j=$ $1, \ldots, n$, are all coherent risk measures (for the definition and related proposition of coherent risk measures, see Dhaene et al. [25]), $\mathbf{Z}^{*}$ achieves Pareto optimum if and only if $\mathbf{Z}^{*}$ is also the solution to the optimization problem

$$
\min _{\mathbf{Z} \in D_{n}(Y)}\left\{\sum_{j=1}^{n} \rho_{j}\left(Z_{j}\right)\right\} .
$$

Since now the problem involves multiple reinsurers, we need to redefine the risks undertaken by the insurers and reinsurers after reinsurance. After the insurer cedes the risk $X$ to several reinsurers, the deductible of the insurer is transferred into $T^{\mathrm{f}}(X)$ which is equivalent to

$$
\begin{aligned}
T^{\mathbf{f}}(X) & \triangleq R_{\mathbf{f}}(X)+\sum_{j=1}^{m} \Pi_{j}\left(f_{j}(X)\right) \\
& =X-\sum_{j=1}^{m} f_{j}(X)+\sum_{j=1}^{m} \Pi_{j}\left(f_{j}(X)\right) .
\end{aligned}
$$

On the other hand, the $j$ th reinsurer undertakes a total risk of $\widetilde{T}_{j}^{f_{j}}(X)$ which can be calculated by

$$
\widetilde{T}_{j}^{f_{j}}(X) \triangleq f_{j}(X)-\Pi_{j}\left(f_{j}(X)\right), \quad j=1, \ldots, m .
$$


It is easy to observe that $T^{\mathbf{f}}(X)+\sum_{j=1}^{m} \widetilde{T}_{j}^{f_{j}}(X)=X$; hence, we are able to use the ceded-loss function $f_{j}$ to split the risk $X$, so as to make the reinsurance market achieve Pareto optimum.

Next, assume that the insurers and all reinsurers adopt coherent distortion risk measures, which can be denoted as $\rho_{G_{R}}(\cdot)$ and $\rho_{G_{j}}(\cdot), j=1, \ldots, m$, respectively. Furthermore, suppose that the generating functions $G_{R}$ and $G_{j}$ of the distortion risk measures are continuous. Based upon optimization problem (6), we solve for

$$
\begin{aligned}
& \min _{\mathbf{f} \in \mathscr{G}_{m}}\left\{\rho_{G_{R}}\left(T^{\mathbf{f}}(X)\right)+\sum_{j=1}^{m} \rho_{G_{R}}\left(\widetilde{T}_{j}^{f_{j}}(X)\right)\right\} \\
= & \min _{\mathbf{f} \in \mathscr{G}_{m}}\left\{\rho_{G_{R}}\left(R_{\mathbf{f}}(X)\right)+\sum_{j=1}^{m} \rho_{G_{j}}\left(f_{j}(X)\right)\right\} .
\end{aligned}
$$

Consequently, the solution to problem (9) achieves Pareto optimum.

Before analyzing the models above, the definitions and notations of some set are given, which will help us prove the relevant issues and provide follow-up solutions. Denote sets

$$
\begin{aligned}
& \Omega_{j}^{-} \triangleq\left\{x \in[0,1] \mid G_{j}(x)<\widetilde{G}_{j}(x)\right\}, \quad j=1, \ldots, m ; \\
& \Omega_{j}^{0} \triangleq\left\{x \in[0,1] \mid G_{j}(x)=\widetilde{G}_{j}(x)\right\}, \quad j=1, \ldots, m ; \\
& \Omega_{R}^{-} \triangleq\left\{x \in[0,1] \mid G_{R}(x)<\widetilde{G}_{R}(x)\right\} ; \\
& \Omega_{R}^{0} \triangleq\left\{x \in[0,1] \mid G_{R}(x)=\widetilde{G}_{R}(x)\right\},
\end{aligned}
$$

where $\widetilde{G}_{R}(x) \triangleq \min \left\{G_{1}(x), \ldots, G_{m}(x)\right\}, \widetilde{G}_{j}(x) \triangleq \min \left\{G_{R}(x)\right.$, $\left.G_{1}(x), \ldots, G_{j-1}(x), G_{j+1}(x), G_{j+1}(x), \ldots, G_{m}(x)\right\}$. According to the definitions above, we have

$$
\bigcup_{j=1}^{m}\left(\Omega_{j}^{-} \cup \Omega_{j}^{0}\right) \cup\left(\Omega_{R}^{-} \cup \Omega_{R}^{0}\right)=[0,1] .
$$

Proposition 3. Each $\Omega_{j}^{-}, \Omega_{j}^{0}, \Omega_{R}^{-}$, and $\Omega_{R}^{0}$ is able to be written in the following forms, that is, unions of mutually disjoint intervals:

$$
\begin{aligned}
\Omega_{j}^{-} & =\bigcup_{j=1}^{m_{j}^{-}}\left(q_{j}^{-}(k), q_{j}^{+}(k)\right), \quad j=1, \ldots, m ; \\
\Omega_{j}^{0} & =\bigcup_{k=1}^{m_{j}^{0}}\left[r_{j}^{-}(k), r_{j}^{+}(k)\right], \quad j=1, \ldots, m ; \\
\Omega_{R}^{-} & =\bigcup_{k=1}^{m_{R}^{-}}\left(q_{R}^{-}(k), q_{R}^{+}(k)\right) ; \\
\Omega_{R}^{0} & =\bigcup_{k=1}^{m_{R}^{0}}\left[r_{R}^{-}(k), r_{R}^{+}(k)\right], \\
\bigcup_{R}^{0} \Omega_{j}^{0} \cup \Omega_{R}^{0} & =\bigcup_{k=1}^{m^{0}}\left[r^{-}(k), r^{+}(k)\right],
\end{aligned}
$$

where $m_{j}^{-}, m_{j}^{0}, m_{R}^{-}, m_{R}^{0}, m^{0} \in\left\{\mathbb{N}^{+} \cup\{+\infty\}\right.$.
Proof. Firstly, for any rational number satisfying $j=1, \ldots, m$, there exists $\delta_{j}^{-}>0$ such that $G_{j}(y)<\widetilde{G}_{j}(y)$ for any $y \in\left[x_{j}^{-}\right.$ $\left.\delta_{j}^{-}, x_{j}^{-}\right]$, since $G_{j}$ is continuous. We define

$$
\begin{aligned}
& q_{j}^{-}\left(x_{j}^{-}\right)=\inf \left\{u_{j}: x_{j}^{-} \in\left[u_{j}, v_{j}\right], G_{j}(y)\right. \\
& \left.<\widetilde{G}_{j}(y), \forall y \in\left[u_{j}, v_{j}\right]\right\}, \\
& q_{j}^{+}\left(x_{j}^{-}\right)=\sup \left\{v_{j}: x_{j}^{-} \in\left[u_{j}, v_{j}\right], G_{j}(y)\right. \\
& \left.<\widetilde{G}_{j}(y), \forall y \in\left[u_{j}, v_{j}\right]\right\} .
\end{aligned}
$$

According to the continuity of $G_{j}$, it is easy to observe that

$$
\begin{aligned}
& G_{j}\left(q_{j}^{-}\left(x_{j}^{-}\right)\right)=\widetilde{G}_{j}\left(q_{j}^{-}\left(x_{j}^{-}\right)\right)=0 ; \\
& G_{j}\left(q_{j}^{+}\left(x_{j}^{-}\right)\right)=\widetilde{G}_{j}\left(q_{j}^{+}\left(x_{j}^{-}\right)\right)=0 .
\end{aligned}
$$

Additionally, for any $y \in\left(q_{j}^{-}\left(x_{j}^{-}\right), q_{j}^{+}\left(x_{j}^{-}\right)\right)$, we get $G_{j}(y)<\widetilde{G}_{j}(y)$. So $\Omega_{j}^{-}$is able to be written as $\Omega_{j}^{-}=$ $\bigcup_{\left\{x \in[0,1], G_{j}(x)<\widetilde{G}_{j}(x)\right\}}\left(q_{j}^{-}(x), q_{j}^{+}(x)\right)$. Based on the denseness and countability of rational number $x_{j}^{-}$in $[0,1]$, we find mutually disjoint sets $\left(q_{j}^{-}(k), q_{j}^{+}(k)\right)$ such that (12) holds.

Now let us analyze the set $\Omega_{j}^{0}$. For any $x_{j}^{0}$ satisfying $G_{j}\left(x_{j}^{0}\right)=\widetilde{G}_{j}\left(x_{j}^{0}\right), j=1, \ldots, m$, we define

$$
\begin{aligned}
& r_{j}^{-}\left(x_{j}^{0}\right)=\inf \left\{u_{j}: x_{j}^{0} \in\left[u_{j}, v_{j}\right], G_{j}(y)\right. \\
& \left.=\widetilde{G}_{j}(y), \forall y \in\left[u_{j}, v_{j}\right]\right\}, \\
& r_{j}^{+}\left(x_{j}^{0}\right)=\inf \left\{v_{j}: x_{j}^{0} \in\left[u_{j}, v_{j}\right], G_{j}(y)\right. \\
& \left.=\widetilde{G}_{j}(y), \forall y \in\left[u_{j}, v_{j}\right]\right\} .
\end{aligned}
$$

Similarly, (13) holds. The expressions for $\Omega_{R}^{-}$and $\Omega_{R}^{0}$ as in (14) and (15) can be constructed similarly. At last, for (16), we only need to sort the interval end points $r_{j}^{-}(k), r_{j}^{+}(k), r_{R}^{-}(k)$ and $r_{R}^{+}(k)$ in increasing sequence and finally eliminate the equal ones.

\section{Optimal Ceded-Loss Functions}

Now we construct the ceded-loss functions which can achieve the Pareto optimum.

Theorem 4. Assume that all generation functions $G_{R}$ and $G_{j}$, $j=1, \ldots, m$, for the distortion risk measures are continuous; 
then, the optimal solution $\mathbf{f}^{*}=\left(f_{1}^{*}, \ldots, f_{m}^{*}\right)$ and $R_{\mathbf{f}}^{*}$ to problem (9) are

$$
\begin{aligned}
& f_{j}^{*}(x)=\sum_{k=1}^{m_{j}^{-}}\left[\left(x-S_{X}^{(-1)}\left(q_{j}^{+}(k)\right)\right)_{+}\right. \\
& \left.-\left(x-S_{X}^{(-1)}\left(q_{j}^{-}(k)\right)\right)_{+}\right] \\
& +\sum_{k=1}^{m_{j}^{0}} l_{k, j}\left[\left(x-S_{X}^{(-1)}\left(r_{j}^{+}(k)\right)\right)_{+}\right. \\
& \left.-\left(x-S_{X}^{(-1)}\left(r_{j}^{-}(k)\right)\right)_{+}\right], \\
& R_{\mathbf{f}}^{*}(x)=\sum_{k=1}^{m_{R}^{-}}\left[\left(x-S_{X}^{(-1)}\left(q_{R}^{+}(k)\right)\right)_{+}\right. \\
& \left.-\left(x-S_{X}^{(-1)}\left(q_{R}^{-}(k)\right)\right)_{+}\right] \\
& +\sum_{k=1}^{m_{R}^{0}} l_{k, R}\left[\left(x-S_{X}^{(-1)}\left(r_{R}^{+}(k)\right)\right)_{+}\right. \\
& \left.-\left(x-S_{X}^{(-1)}\left(r_{R}^{-}(k)\right)\right)_{+}\right],
\end{aligned}
$$

$$
\min _{\mathbf{f} \in \mathscr{S}_{m}}\left\{\rho_{G_{R}}\left(T^{\mathrm{f}}(X)+\sum_{j=1}^{m} \rho_{G_{j}}\left(\widetilde{T}_{j}^{f_{j}}(X)\right)\right\}\right)=\int_{0}^{+\infty} \min \left\{G_{R}\left(S_{X}(t)\right), G_{1}\left(S_{X}(t)\right), \ldots, G_{m}\left(S_{X}(t)\right)\right\} \mathrm{d} t .
$$

Proof. First we explain that the numbers $l_{k, R}$ and $l_{k, j}$ satisfying (22) exist. According to the divisions of $\Omega_{R}^{0}$ and $\Omega_{j}^{0}, j=$ $1, \ldots, m$, in Proposition 3, every $\left[r^{-}(k), r^{+}(k)\right]$ consisted of the unions of $\left[r_{j}^{-}(k), r_{j}^{+}(k)\right]$ and $\left[r_{R}^{-}(k), r_{R}^{+}(k)\right]$. Hence, for any $\left[r_{R}^{-}(k), r_{R}^{+}(k)\right]$, it is easy to find out that $\left[r_{R}^{-}(k), r_{R}^{+}(k)\right]$ must be in some interval $\left[r^{-}\left(k^{\prime}\right), r^{+}\left(k^{\prime}\right)\right], k^{\prime}=1, \ldots, m^{0}$. Therefore, without loss of generality, we assume that there exist several intervals $\left[r_{j_{0}}^{-}\left(k_{0}\right), r_{j_{0}}^{+}\left(k_{0}\right)\right]$ satisfying

$$
\begin{aligned}
{\left[r^{-}\left(k^{\prime}\right), r^{+}\left(k^{\prime}\right)\right]=} & {\left[r_{j_{0}}^{-}\left(k_{0}\right), r_{j_{0}}^{+}\left(k_{0}\right)\right] } \\
& \cup\left[r_{R}^{-}(k), r_{R}^{+}(k)\right] ; \\
r_{R}^{-}(k) \leq & r_{j_{0}}^{+}\left(k_{0}\right) \leq r_{R}^{+}(k)=r^{+}\left(k^{\prime}\right) ; \\
r^{-}\left(k^{\prime}\right)= & r_{j_{0}}^{+}\left(k_{0}\right),
\end{aligned}
$$

where $j_{0} \in\{1, \ldots, m\}, k_{0} \in\left\{1, \ldots, m_{j_{0}}^{0}\right\}$.

On the other hand, according to the definition of $\Omega_{R}^{0}$, there exists at least one function $G_{j}$ on $\left[r_{j_{0}}^{+}\left(k_{0}\right), r_{R}^{+}(k)\right]$ such that $G_{j}(x)=G_{R}(x)$. Therefore, we assume that there exist $m^{\prime}$ functions $\left(1 \leq m^{\prime} \leq m\right), G_{k_{1}}, \ldots, G_{k_{m}},\left(1 \leq k_{1} \leq k_{m^{\prime}} \leq m\right)$ on $\left[r_{j_{0}}^{+}\left(k_{0}\right), r_{R}^{+}(k)\right]$ such that

$$
G_{R}(x)=G_{k_{1}}(x)=\cdots=G_{k_{m^{\prime}}}(x) .
$$

where $S_{X}^{(-1)}(y) \triangleq \inf \left\{x: S_{X}(x) \leq y\right\}$, and the constants $l_{k, R}, l_{k, j} \geq 0$ satisfy

$$
\begin{aligned}
& \sum_{j=1}^{m} \sum_{k=1}^{m_{j}^{0}} l_{k, j}\left[\left(x-S_{X}^{(-1)}\left(r_{j}^{+}(k)\right)\right)_{+}\right. \\
& \left.-\left(x-S_{X}^{(-1)}\left(r_{j}^{-}(k)\right)\right)_{+}\right]+\sum_{k=1}^{m_{R}^{0}} l_{k, R}[(x \\
& \left.\left.-S_{X}^{(-1)}\left(r_{R}^{+}(k)\right)\right)_{+}-\left(x-S_{X}^{(-1)}\left(r_{R}^{-}(k)\right)\right)_{+}\right]=\sum_{j=1}^{m}[(x \\
& \left.\left.-S_{X}^{(-1)}\left(r^{+}(k)\right)\right)_{+}-\left(x-S_{X}^{(-1)}\left(r^{-}(k)\right)\right)_{+}\right], \\
& \forall x \in[0,+\infty] .
\end{aligned}
$$

Moreover, the minimum is

Corresponding to each function above, we need to find out the nonnegative constants $l_{k, R}$ and $l_{k, k_{j}}, j=1, \ldots, m^{\prime}$, satisfying the following equations:

$$
\begin{aligned}
& l_{k, R}+\sum_{j=1}^{m^{\prime}} l_{k, k_{j}}=1, \\
& l_{k, R}\left[\left(x-S_{X}^{(-1)}\left(r_{j_{0}}^{+}\left(k_{0}\right)\right)\right)_{+}-\left(x-S_{X}^{(-1)}\left(r_{R}^{+}(k)\right)\right)_{+}\right] \\
& \quad+\sum_{j=1}^{m^{\prime}} l_{k, k_{j}} \\
& \cdot\left[\left(x-S_{X}^{(-1)}\left(r_{j_{0}}^{+}\left(k_{0}\right)\right)\right)_{+}-\left(x-S_{X}^{(-1)}\left(r_{R}^{+}(k)\right)\right)_{+}\right] \\
& =\left(l_{k, R}+l_{A}\right) \\
& \cdot\left[\left(x-S_{X}^{(-1)}\left(r_{j_{0}}^{+}\left(k_{0}\right)\right)\right)_{+}-\left(x-S_{X}^{(-1)}\left(r_{R}^{+}(k)\right)\right)_{+}\right] \\
& =\left(x-S_{X}^{(-1)}\left(r_{j_{0}}^{+}\left(k_{0}\right)\right)\right)_{+}-\left(x-S_{X}^{(-1)}\left(r_{R}^{+}(k)\right)\right)_{+},
\end{aligned}
$$

where $l_{A}=\sum_{j=1}^{m^{\prime}} l_{k, k_{j}} \geq 0$. For $\left[r_{R}^{-}(k), r_{j_{0}}^{+}\left(k_{0}\right)\right]$ and $l_{k, R}$ in (26), it is necessary to calculate the constants $l_{k, j_{0}}$ and $l_{B}$ such that

$$
l_{k, R}+l_{k, j_{0}}+l_{B}=1
$$


The definition of $l_{B}$ is the same as $l_{A}$. Notice that if there exist only two functions $G_{j_{0}}$ and $G_{R}$ on $\left[r_{R}^{-}(k), r_{j_{0}}^{+}\left(k_{0}\right)\right]$ equal to each other, then $l_{B} \equiv 0$. Similarly, we have

$$
l_{k, j_{0}}+l_{C}=1
$$

in the interval $\left[r_{j_{0}}^{-}\left(k_{0}\right), r_{R}^{-}(k)\right]$.

Next, combining (26), (28), and (29), we show the existence of nonnegative solutions to the equations. In fact, when $l_{A}=l_{k, j_{0}}, l_{B}=0$, and $l_{C}=l_{k, R}$ hold, then $\left[r_{j_{0}}^{-}\left(k_{0}\right)\right.$, $\left.r_{j_{0}}^{+}\left(k_{0}\right)\right]=\left[r_{R}^{-}(k), r_{R}^{+}(k)\right]$. Meanwhile, the equations reduce to one equation; that is, $l_{k, R}+l_{k, j_{0}}=1$. Clearly, there exist nonnegative solutions. Besides, it can be observed that "the number of unknowns $\geq$ the rank of parameter matrix," so we deduce the existence of nonnegative solutions.

In addition, (27) implies

$$
\begin{aligned}
& \left(l_{k, R}+l_{A}\right) \\
& \cdot\left[\left(x-S_{X}^{(-1)}\left(r_{j_{0}}^{+}\left(k_{0}\right)\right)\right)_{+}-\left(x-S_{X}^{(-1)}\left(r_{R}^{+}(k)\right)\right)_{+}\right] \\
& +\left(l_{k, R}+l_{k, j_{0}}+l_{B}\right) \\
& \cdot\left[\left(x-S_{X}^{(-1)}\left(r_{R}^{-}(k)\right)\right)_{+}-\left(x-S_{X}^{(-1)}\left(r_{j_{0}}^{+}\left(k_{0}\right)\right)\right)_{+}\right] \\
& \quad+\left(l_{k, j_{0}}+l_{C}\right) \\
& \cdot\left[\left(x-S_{X}^{(-1)}\left(r_{j_{0}}^{-}\left(k_{0}\right)\right)\right)_{+}-\left(x-S_{X}^{(-1)}\left(r_{R}^{-}(k)\right)\right)_{+}\right] \\
& \quad=\left(x-S_{X}^{(-1)}\left(r^{-}\left(k^{\prime}\right)\right)\right)_{+}-\left(x-S_{X}^{(-1)}\left(r^{+}\left(k^{\prime}\right)\right)\right)_{+} .
\end{aligned}
$$

We get $l_{k, j}$ and $l_{k, R}$ based on the equation above. Then, based upon (16), we have demonstrated that there exist the constants $l_{k, R}$ and $l_{k, j}$ satisfying (22).

Next, the risk measures will be transformed in the following form which can be handled more easily. Recalling that $T^{\mathrm{f}}(X)+\sum_{j=1}^{m} \widetilde{T}_{j}^{f_{j}}(X)=X$, we know the copula of random vector $\left(T^{\mathrm{f}}(X), \widetilde{T}_{1}^{f_{1}}(X), \ldots, \widetilde{T}_{m}^{f_{m}}(X)\right)$ achieves the FrechetHoeffding upper bound. Thus, $\left(T^{\mathbf{f}}(X), \widetilde{T}_{1}^{f_{1}}(X), \ldots, \widetilde{T}_{m}^{f_{m}}(X)\right)$ is called "comonotonic" random vector.

Since the coherent risk measures have the property of "additivity for the comonotonic random vector" (for the definition and related proposition of coherent risk measures, see Section 5.1 in Dhaene et al. [25] and Theorem 3.3 in Cai and Wei [26]), we conclude that

$$
\begin{aligned}
& \rho_{G_{R}}\left(T^{\mathrm{f}}(X)\right)+\sum_{j=1}^{m} \rho_{G_{j}}\left(\widetilde{T}_{j}^{f_{j}}(X)\right) \\
& =\rho_{G_{R}}\left(R_{\mathbf{f}}(X)\right)+\sum_{j=1}^{m} \rho_{G_{j}}\left(f_{j}(X)\right) \\
& =\rho_{G_{R}}(X)+\sum_{j=1}^{m}\left[\rho_{G_{j}}\left(f_{j}(X)\right)-\rho_{G_{R}}\left(f_{j}(X)\right)\right] .
\end{aligned}
$$

Denote $f_{j}^{(-1)}(y)=\inf \left\{x: f_{j}(x)>y\right\}$; it can be verified that

$$
\mathbb{P}\left(f_{j}(x)>y\right)=\mathbb{P}\left(X>f_{j}^{(-1)}(y)\right), \quad \forall y \geq 0 .
$$

Hence, (31) is rewritten as

$$
\begin{aligned}
& \rho_{G_{R}}(X)+\sum_{j=1}^{m} \int_{0}^{+\infty} G_{j}\left(\mathbb{P}\left(f_{j}(x)>t\right)\right) \\
& -G_{R}\left(\mathbb{P}\left(f_{j}(x)>t\right)\right) \mathrm{d} t=\rho_{G_{R}}(X) \\
& +\sum_{j=1}^{m} \int_{0}^{+\infty} G_{j}\left(\mathbb{P}\left(X>f_{j}^{(-1)}(t)\right)\right) \\
& -G_{R}\left(\mathbb{P}\left(X>f_{j}^{(-1)}(t)\right)\right) \mathrm{d} t=\rho_{G_{R}}(X) \\
& +\sum_{j=1}^{m} \int_{0}^{+\infty} G_{j}\left(S_{X}\left(f_{j}^{(-1)}(t)\right)\right) \\
& -G_{R}\left(S_{X}\left(f_{j}^{(-1)}(t)\right)\right) \mathrm{d} t=\rho_{G_{R}}(X) \\
& +\sum_{j=1}^{m} \int_{\mathbb{R}^{+}}\left(G_{j} \circ f_{j}^{(-1)}-G_{R} \circ f_{j}^{(-1)}\right) \mathrm{d} \mu,
\end{aligned}
$$

where $\mu$ is a Lebesgue measure. According to the continuity of $f_{j}$, there exists another measure $v$ satisfying the following general integrals:

$$
\begin{gathered}
\int_{\mathbb{R}^{+}}\left(G_{j} \circ f_{j}^{(-1)}-G_{R} \circ f_{j}^{(-1)}\right) \mathrm{d} \mu=\int_{\mathbb{R}^{+}}\left(G_{j}-G_{R}\right) \mathrm{d} v \\
=\int_{\mathbb{R}^{+}}\left[G_{j}\left(S_{X}(t)\right)-G_{R}\left(S_{X}(t)\right)\right] \mathrm{d} f_{j}(t) .
\end{gathered}
$$

Now we analyze each item in (33). Expression (20) means the following equation holds:

$$
\begin{aligned}
\sum_{j=1}^{m} & \int_{\mathbb{R}^{+}}\left[G_{j}\left(S_{X}(t)\right)-G_{R}\left(S_{X}(t)\right)\right] \mathrm{d} f_{j}^{*}(t) \\
= & \sum_{j=1}^{m} \int_{\left\{S_{X}(t) \in \Omega_{j}^{-}\right\}}\left[G_{j}\left(S_{X}(t)\right)-G_{R}\left(S_{X}(t)\right)\right] \mathrm{d} t \\
& +\sum_{j=1}^{m} \sum_{k=1}^{m_{j}^{0}} l_{k, j} \\
& \cdot \int_{S_{X}^{(-1)}\left(r_{j}^{+}(k)\right)}^{S_{X}^{(-1)}\left(r_{j}^{-}(k)\right)}\left[G_{j}\left(S_{X}(t)\right)-G_{R}\left(S_{X}(t)\right)\right] \mathrm{d} t .
\end{aligned}
$$

For any $\left[r_{R}^{-}(k), r_{R}^{+}(k)\right]$, there exists at least one $G_{k_{i}}\left(k_{i} \in\right.$ $\{1, \ldots, m\})$ satisfying $G_{k_{i}}\left(S_{X}(t)\right)=G_{R}\left(S_{X}(t)\right)$. Consequently, there exist $m_{k}^{\prime}$ functions in $G_{1}, \ldots, G_{m}$ such that

$$
\begin{aligned}
& G_{k_{1}}(x)=\cdots=G_{k_{m_{k}^{\prime}}}(x)=G_{R}(x) \\
& x \in\left[r_{R}^{-}(k), r_{R}^{+}(k)\right] .
\end{aligned}
$$


Let us recall the procedure for finding the constants $l_{k, R}$ and $l_{k, j}$. Finally, we have

$$
\begin{aligned}
& \int_{\left\{S_{X}(t) \in \bigcup_{j=1}^{m} \Omega_{j}^{0} \cup \Omega_{R}^{0}\right\}} G_{R}\left(S_{X}(t)\right) \mathrm{d} t+\sum_{j=1}^{m} \sum_{k=1}^{m_{j}^{0}} l_{k, j} \\
& \cdot \int_{S_{X}^{(-1)}\left(r_{j}^{+}(k)\right)}^{S_{X}^{(-1)}\left(r_{j}^{-}(k)\right)}\left[G_{j}\left(S_{X}(t)\right)-G_{R}\left(S_{X}(t)\right)\right] \mathrm{d} t \\
& =\int_{\left\{S_{X}(t) \in \bigcup_{j=1}^{m} \Omega_{j}^{0} \cup \Omega_{R}^{0}\right\}} G_{R}\left(S_{X}(t)\right) \mathrm{d} t+\sum_{j=1}^{m} \sum_{k=1}^{m_{j}^{0}} l_{k, j} \\
& \cdot \int_{S_{X}^{(-1)}\left(r_{j}^{+}(k)\right)}^{S_{X}^{(-1)}\left(r_{j}^{-}(k)\right)}\left[G_{j}\left(S_{X}(t)\right)-G_{R}\left(S_{X}(t)\right)\right] \mathrm{d} t \\
& +\sum_{k=1}^{m_{R}^{0}} l_{k, R} \sum_{i=1}^{m_{k}^{\prime}} \int_{S_{X}^{(-1)}\left(r_{j}^{+}(k)\right)}^{S_{X}^{(-1)}\left(r_{j}^{-}(k)\right)}\left[G_{k_{i}}\left(S_{X}(t)\right)\right. \\
& \left.-G_{R}\left(S_{X}(t)\right)\right] \mathrm{d} t \\
& =\int_{\left\{S_{X}(t) \in \bigcup_{j=1}^{m} \Omega_{j}^{0} \cup \Omega_{R}^{0}\right\}} G_{R}\left(S_{X}(t)\right) \mathrm{d} t \\
& +\int_{\left\{S_{X}(t) \in \bigcup_{j=1}^{m} \Omega_{j}^{0} \cap \Omega_{R}^{0}\right\}}\left[G_{R}\left(S_{X}(t)\right)\right. \\
& \left.-G_{R}\left(S_{X}(t)\right)\right] \mathrm{d} t \\
& +\int_{\left\{S_{X}(t) \in \bigcup_{j=1}^{m} \Omega_{j}^{0} / \Omega_{R}^{0}\right\}}\left[\widetilde{G}_{R}\left(S_{X}(t)\right)\right. \\
& \left.-G_{R}\left(S_{X}(t)\right)\right] \mathrm{d} t \\
& =\int_{\left\{S_{X}(t) \in \bigcup_{j=1}^{m} \Omega_{j}^{0} \cup \Omega_{R}^{0}\right\}} \min \left\{G_{R}\left(S_{X}(t)\right), G_{1}\left(S_{X}(t)\right),\right. \\
& \left.\ldots, G_{m}\left(S_{X}(t)\right)\right\} \mathrm{d} t \text {. }
\end{aligned}
$$

Furthermore, $\rho_{G_{R}}(X)$ can be rewritten as

$$
\begin{aligned}
& \rho_{G_{R}}(X)=\int_{0}^{+\infty} G_{R}\left(S_{X}(t)\right) \mathrm{d} t \\
& =\sum_{j=1}^{m} \int_{\left\{S_{X}(t) \in \Omega_{j}^{-}\right\}} G_{R}\left(S_{X}(t)\right) \mathrm{d} t \\
& \quad+\int_{\left\{S_{X}(t) \in \Omega_{R}^{-}\right\}} G_{R}\left(S_{X}(t)\right) \mathrm{d} t \\
& +\int_{\left\{S_{X}(t) \in \cup_{j=1}^{m} \Omega_{j}^{0} \cup \Omega_{R}^{0}\right\}} G_{R}\left(S_{X}(t)\right) \mathrm{d} t .
\end{aligned}
$$

Combining (35), (37), and (38), we have the following equations as the conclusion:

$$
\begin{aligned}
& \rho_{G_{R}}\left(T^{\mathrm{f}^{*}}(X)\right)+\sum_{j=1}^{m} \rho_{G_{j}}\left(\widetilde{T}_{j}^{f_{j}^{*}}(X)\right)=\rho_{G_{R}}(X) \\
& +\sum_{j=1}^{m} \int_{\left\{S_{X}(t) \in \Omega_{j}^{-}\right\}}\left[G_{j}\left(S_{X}(t)\right)-G_{R}\left(S_{X}(t)\right)\right] \mathrm{d} t
\end{aligned}
$$

$$
\begin{aligned}
& +\sum_{j=1}^{m} \sum_{k=1}^{m_{j}^{0}} l_{k, j} \int_{S_{X}^{(-1)}\left(r_{j}^{+}(k)\right)}^{S_{X}^{(-1)}\left(r_{j}^{-}(k)\right)}\left[G_{j}\left(S_{X}(t)\right)\right. \\
& \left.-G_{R}\left(S_{X}(t)\right)\right] \mathrm{d} t \\
& =\sum_{j=1}^{m} \int_{\left\{S_{X}(t) \in \Omega_{j}^{-}\right\}} G_{j}\left(S_{X}(t)\right) \mathrm{d} t \\
& +\int_{\left\{S_{X}(t) \in \Omega_{R}^{-}\right\}} G_{R}\left(S_{X}(t)\right) \mathrm{d} t \\
& +\int_{\left\{S_{X}(t) \in \bigcup_{k=1}^{m} \Omega_{j}^{0} \cup \Omega_{R}^{0}\right\}} \min \left\{G_{R}\left(S_{X}(t)\right), G_{1}\left(S_{X}(t)\right),\right. \\
& \left.\ldots, G_{m}\left(S_{X}(t)\right)\right\} \mathrm{d} t=\int_{0}^{+\infty} \min \left\{G_{R}\left(S_{X}(t)\right),\right. \\
& \left.G_{1}\left(S_{X}(t)\right), \ldots, G_{m}\left(S_{X}(t)\right)\right\} \mathrm{d} t .
\end{aligned}
$$

Then, for any $f_{j} \in \mathscr{G}_{m}$, we get

$$
\begin{aligned}
\rho_{G_{R}} & \left(T^{\mathrm{f}}(X)\right)+\sum_{j=1}^{m} \rho_{G_{j}}\left(\widetilde{T}_{j}^{f_{j}}(X)\right) \\
= & \rho_{G_{R}}(X)+\sum_{j=1}^{m}\left(\rho_{G_{j}}\left(f_{j}(X)\right)-\rho_{G_{R}}\left(f_{j}(X)\right)\right) .
\end{aligned}
$$

When $S_{X}(t) \in \Omega_{j}^{-} \cup \Omega_{j}^{0}$, it can be seen that $G_{j}\left(S_{X}(t)\right)-$ $G_{R}\left(S_{X}(t)\right) \leq 0$. Recall the Lipschitz continuous of $R_{\mathrm{f}}$ and every $f_{j}, j=1, \ldots, m$; expression (40) is demonstrated as the following form:

$$
\begin{aligned}
& \rho_{G_{R}}(X)+\sum_{j=1}^{m}\left(\rho_{G_{j}}\left(f_{j}(X)\right)-\rho_{G_{R}}\left(f_{j}(X)\right)\right) \\
& \geq \rho_{G_{R}}(X)+\sum_{j=1}^{m} \int_{\left\{S_{X}(t) \in \Omega_{j}^{-} \cup \Omega_{j}^{0}\right\}}\left[G_{j}\left(S_{X}(t)\right)\right. \\
& \left.\quad-G_{R}\left(S_{X}(t)\right)\right] \mathrm{d} f_{j}(t) \geq \rho_{G_{R}}(X) \\
& +\sum_{j=1}^{m} \int_{\left\{S_{X}(t) \in \Omega_{j}^{-} \cup \Omega_{j}^{0}\right\}}\left[G_{j}\left(S_{X}(t)\right)-G_{R}\left(S_{X}(t)\right)\right] \mathrm{d} t \\
& =\int_{0}^{+\infty} \min \left\{G_{R}\left(S_{X}(t)\right), G_{1}\left(S_{X}(t)\right), \ldots,\right. \\
& \left.G_{m}\left(S_{X}(t)\right)\right\} \mathrm{d} t .
\end{aligned}
$$

This means $\int_{0}^{+\infty} \min \left\{G_{R}\left(S_{X}(t)\right), G_{1}\left(S_{X}(t)\right), \ldots, G_{m}\left(S_{X}(t)\right)\right\} \mathrm{d} t$ is equal to the lower bound of

$$
\rho_{G_{R}}\left(T^{\mathrm{f}}(X)\right)+\sum_{j=1}^{m} \rho_{G_{j}}\left(\widetilde{T}_{j}^{f_{j}}(X)\right) .
$$

Finally, based on (39), (23) holds definitely. 
Intuitively, when the generating functions are small, the corresponding distortion risk measures are also small; that is, the risk levels are relatively lower. The theorem above shows that various participants in the reinsurance market only bare the loss when their risk measures are on the "smallest intervals," and the reinsurance market achieves the Pareto optimum in such a situation. More specifically, (20) shows that the $j$ th reinsurer should bare the loss under every interval $\left[S_{X}^{(-1)}\left(q_{j}^{+}(k)\right), S_{X}^{(-1)}\left(q_{j}^{-}(k)\right)\right]$ since the corresponding generation function is strictly less than other generators under $\left[S_{X}^{(-1)}\left(q_{j}^{+}(k)\right), S_{X}^{(-1)}\left(q_{j}^{-}(k)\right)\right]$. However, for the loss in the interval $\left[S_{X}^{(-1)}\left(q_{j}^{+}(k)\right), S_{X}^{(-1)}\left(q_{j}^{-}(k)\right)\right]$, at least there exists another reinsurer or the insurer which uses the same generation function as the $j$ th reinsurer. Therefore, the risk divisions in such intervals can be arbitrary but the nonnegative constants $l_{k, R}$ and every $l_{k, j}$ need to satisfy (22). Meanwhile, the retention part (21) is able to be explained similarly.
At last, we apply two numerical examples to show how to find the optimal reinsurance functions under the Pareto optimum, which may help readers have a better understanding of our work.

\section{Numerical Examples}

Example 1. Assume that the loss $X$ follows an exponential distribution with mean $\lambda$ before reinsurance; that is, the CDF of $X$ is $F_{X}(x)=1-\exp \{x / \lambda\}, \lambda=1000$. Moreover, the insurer adopts the risk measure in the following form:

$$
\begin{array}{r}
\rho_{G_{R}}(Y)=\int_{0}^{+\infty} \min \left\{\frac{S_{Y}(t)}{\alpha}, 1\right\} \mathrm{d} t, \\
\quad \alpha=0.1, \forall Y \in \mathcal{X} .
\end{array}
$$

Suppose that $X$ is ceded to two reinsurers, and the ceded-loss functions are denoted as $f_{1}$ and $f_{2}$, respectively. Meanwhile, the corresponding risk measures are

$$
\begin{aligned}
& \rho_{G_{1}}(Y)=\int_{0}^{+\infty} \min \left\{\max \left\{\left(S_{Y}(t)\right)^{q},\left(S_{Y}(t)+b\right) I\left\{S_{Y}(t)>q^{1 /(1-q)}\right\}\right\}, 1\right\} \mathrm{d} t, \\
& q=\frac{1}{6}, \quad b=q^{q /(1-q)}-q^{1 /(1-q)}=0.582, \forall Y \in \mathscr{X}, \\
& \rho_{\mathrm{G}_{2}}(Y)=\int_{0}^{+\infty} \min \left\{\max \left\{\frac{k S_{Y}(t)}{\gamma}, k+(1-k) \cdot \frac{\left(S_{Y}(t)-\gamma\right)}{(\beta-\gamma)}\right\}, 1\right\} \mathrm{d} t, \quad k=0.06, \beta=0.05, \gamma=0.01, \forall Y \in \mathscr{X} .
\end{aligned}
$$

First, we derive the form of the reinsurance function under Pareto optimum. Based on the form of the distortion risk measure above, $\rho_{G_{R}}$ is a CVaR risk measure. For $G_{1}(s)$, $s \in[0,1]$, use $s^{*}$ to denote the solution of equation $s^{q}=s+b$ in $[0,1]$. Plugging in all values, we solve for the solutions to the nonlinear equations in MATLAB using the command "fsolve" and get the unique root $s^{*}=0.117$. In fact, the straight line $s+b$ is the tangent of $s^{q}$ at $s^{*}$. Therefore, because $s^{q}$ is concave, we know $G_{1}$ is also concave. In the following, we

$$
G_{1}(x)-G_{R}(x)= \begin{cases}x^{q}-\frac{x}{\alpha} \geq 0, & 0 \leq x<\alpha^{1 /(1-q)}=0.0631 \\ \max \left\{x^{q},(x+b) I\left\{x>q^{1 /(1-q)}\right\}\right\}-\min \left\{\frac{x}{\alpha}, 1\right\} \leq 0, & \alpha^{1 /(1-q)} \leq x<1-b \\ 0, & 1-b \leq x \leq 1,\end{cases}
$$

consider $G_{3}$. Plugging in $k, \beta$, and $\gamma$, we get $k / \gamma<(1-k) /(\beta-$ $\gamma)$, so $G_{3}$ is not concave. However, $\rho_{G_{2}}(\cdot)$ can be written as

$$
\begin{aligned}
\rho_{G_{2}}(Y)= & \frac{\beta(1-k)}{(\beta-\gamma)} \cdot \operatorname{CVaR}_{\beta}(Y)+\frac{(k \beta-\gamma)}{(\beta-\gamma)} \\
& \cdot \operatorname{CVaR}_{\gamma}(Y),
\end{aligned}
$$

which is the linear combination of the CVaR risk measure. To summarize, $\rho_{G_{1}}(\cdot), \rho_{G_{2}}(\cdot)$, and $\rho_{G_{R}}(\cdot)$ are all coherent risk measures, so the Pareto optimal division problem in (5) is equivalent to the problem in (9).

Next, the exact form of $\Omega_{j}^{-}$and $\Omega_{j}^{0}, j=1,2$, should be found. In step one, we analyze $\Omega_{1}^{-}$and $\Omega_{1}^{0}$, which implies where $G_{1}(x)-G_{R}(x)=0$ holds if and only if $x \in\left\{0, \alpha^{1 /(1-q)}\right\} \cup$ $[1-b, 1]$. For the difference of $G_{1}$ and $G_{2}$, we use $x_{1}^{*}$ to denote the root of $x^{q}=\max \{k x / \gamma, k+(1-k) \cdot(x-\gamma) /(\beta-\gamma)\}$ in $(0,1)$; then, we get the unique root $x_{1}^{*}=0.0313$. Similarly, we have 


$$
G_{1}(x)-G_{2}(x)= \begin{cases}x^{q}-\max \left\{\frac{k x}{\gamma}, k+(1-k) \cdot \frac{(x-\gamma)}{(\beta-\gamma)}\right\} \geq 0, & 0 \leq x<x_{1}^{*} \\ \max \left\{x^{q},(x+b) I\left\{x>q^{1 /(1-q)}\right\}\right\}-\min \left\{k+(1-k) \cdot \frac{(x-\gamma)}{(\beta-\gamma)}, 1\right\} \leq 0, & x_{1}^{*} \leq x<1-b \\ 0, & 1-b \leq x \leq 1 .\end{cases}
$$

And $G_{1}(x)-G_{2}(x)=0$ holds if and only if $x \in\left\{0, x_{1}^{*}\right\} \cup[1-$ $b, 1]$. Then,

$$
\begin{aligned}
& \Omega_{1}^{-}=\left(\alpha^{1 /(1-q)}, 1-b\right) \\
& \Omega_{1}^{0}=\left\{0, \alpha^{1 /(1-q)}\right\} \cup[1-b, 1] .
\end{aligned}
$$

In step two, we get that the unique root of

$$
\min \left\{\frac{x}{\alpha}, 1\right\}=\max \left\{\frac{k x}{\gamma}, k+(1-k) \cdot \frac{(x-\gamma)}{(\beta-\gamma)}\right\}
$$

in $(0,1)$ is $x_{2}^{*}=0.013$. Then, the sets $\Omega_{2}^{-}$and $\Omega_{2}^{0}$ can be built. Thus, we have

$$
G_{2}(x)-G_{R}(x)= \begin{cases}\max \left\{\frac{k x}{\gamma}, k+(1-k) \cdot \frac{(x-\gamma)}{(\beta-\gamma)}\right\}-\frac{x}{\alpha} \leq 0, & 0 \leq x<x_{2}^{*} \\ \min \left\{k+(1-k) \cdot \frac{(x-\gamma)}{(\beta-\gamma)}, 1\right\}-\min \left\{\frac{x}{\alpha}, 1\right\} \geq 0, & x_{2}^{*} \leq x<1-b \\ 0, & 1-b \leq x \leq 1,\end{cases}
$$

where $G_{2}(x)-G_{R}(x)=0$ holds if and only if $x \in\left\{0, x_{2}^{*}\right\} \cup$ $[1-b, 1]$. Therefore,

$$
\begin{aligned}
& \Omega_{2}^{-}=\left(0, x_{2}^{*}\right) \\
& \Omega_{2}^{0}=\left\{0, x_{2}^{*}\right\} \cup[1-b, 1] .
\end{aligned}
$$

Based upon Theorem 4, the form of $f_{1}^{*}, f_{2}^{*}$, and $R_{\mathbf{f}}^{*}$ that achieves Pareto optimum is given as

$$
\begin{aligned}
f_{1}^{*}= & \left(x-S_{X}^{(-1)}(1-b)\right)_{+}-\left(x-S_{X}^{(-1)}\left(\alpha^{1 /(1-q)}\right)\right)_{+} \\
& +l_{1}\left[x-\left(x-S_{X}^{(-1)}(1-b)\right)_{+}\right] \\
= & (x-872.27)_{+}-(x-2763.03)_{+} \\
& +l_{1}\left[x-(x-872.27)_{+}\right] ; \\
f_{2}^{*}= & \left(x-S_{X}^{(-1)}\left(x_{2}^{*}\right)\right)_{+}-\left(x-S_{X}^{(-1)}(0)\right)_{+} \\
& +l_{2}\left[x-\left(x-S_{X}^{(-1)}(1-b)\right)_{+}\right] \\
= & (x-4342.81)_{+}+l_{2}\left[x-(x-872.27)_{+}\right] ; \\
R_{\mathrm{f}}^{*}= & \left(x-S_{X}^{(-1)}\left(\alpha^{1 /(1-q)}\right)\right)_{+}-\left(x-S_{X}^{(-1)}\left(x_{2}^{*}\right)\right)_{+} \\
& +l_{R}\left[x-\left(x-S_{X}^{(-1)}(1-b)\right)_{+}\right] \\
= & (x-2763.03)_{+}-(x-4342.81)_{+} \\
& +l_{R}\left[x-(x-872.27)_{+}\right],
\end{aligned}
$$

where the nonnegative constants $l_{1}, l_{2}, l_{R}$ satisfy $l_{1}+l_{2}+l_{R}=$ 1.
Example 2. In this example, we only replace the distributions of loss based on the example above. Assume that $X$ follows Pareto distribution with CDF:

$$
F_{X}(x)=1-\left(\frac{z_{0}}{\left(x+z_{0}\right)}\right)^{\mu}, \quad \mu=3, z_{0}=2000 .
$$

Similarly, we find the optimal ceded-loss functions as follows:

$$
\begin{aligned}
f_{1}^{*}= & (x-674.88)_{+}-(x-3023.66)_{+} \\
& +l_{1}\left[x-(x-674.88)_{+}\right] \\
f_{2}^{*}= & (x-6505.81)_{+}+l_{2}\left[x-(x-674.88)_{+}\right] ; \\
R_{\mathrm{f}}^{*}= & (x-3023.66)_{+}-(x-6505.81)_{+} \\
& +l_{R}\left[x-(x-674.88)_{+}\right]
\end{aligned}
$$

where the nonnegative constants $l_{1}, l_{2}, l_{R}$ satisfy $l_{1}+l_{2}+l_{R}=$ 1.

This study has an important effect on the risk management and strategic planning of insurance and reinsurance companies and provides guidance for the regulatory authorities to optimize the reinsurance market. In practice, reinsurance should consider some restrictions, such as solvency and default risk. In theory, this paper considers only one kind of Nash equilibrium; thus, more optimal reinsurance strategies can be studied under generalized Nash equilibrium in the future. 


\section{Competing Interests}

The authors declare that there is no conflict of interests regarding the publication of this paper.

\section{Acknowledgments}

This paper was supported by the Fundamental Research Funds for the Central Universities (JBK160101) and the Youth Foundation for Humanities and Social Sciences of Education of China (15YJC630105).

\section{References}

[1] K. Borch, "An attempt to determine the optimum amount of stop loss reinsurance," in Proceedings of the in Transactions of the 16th International Congress of Actuaries, vol. 1, pp. 597-610, 1960.

[2] K. J. Arrow, "Uncertainty and the welfare economics of medical care," American Economic Review, vol. 53, no. 5, pp. 941-973, 1963.

[3] A. Raviv, "The design of an optimal insurance policy," American Economic Review, vol. 69, no. 1, pp. 84-96, 1979.

[4] G. Huberman, D. Mayers, and C. W. Smith Jr., "Optimal insurance policy in demnity schedules," The Bell Journal of Economics, vol. 14, no. 2, pp. 415-426, 1983.

[5] V. R. Young, "Optimal insurance under Wang's premium principle," Insurance: Mathematics and Economics, vol. 25, no. 2, pp. 109-122, 1999.

[6] M. Kaluszka, "Optimal reinsurance under mean-variance premium principles," Insurance: Mathematics and Economics, vol. 28, no. 1, pp. 61-67, 2001.

[7] M. Kaluszka and A. Okolewski, "An extension of Arrow's result on optimal reinsurance contract," The Journal of Risk and Insurance, vol. 75, no. 2, pp. 275-288, 2008.

[8] J. Cai and K. S. Tan, "Optimal retention for a stop-loss reinsurance under the VaR and CTE risk measures," ASTIN Bulletin, vol. 37, no. 1, pp. 93-112, 2007.

[9] J. Cai, K. S. Tan, C. Weng, and Y. Zhang, "Optimal reinsurance under VaR and CTE risk measures," Insurance: Mathematics \& Economics, vol. 43, no. 1, pp. 185-196, 2008.

[10] A. Balbás, B. Balbás, and A. Heras, "Optimal reinsurance with general risk measures," Insurance: Mathematics and Economics, vol. 44, no. 3, pp. 374-384, 2009.

[11] K. S. Tan, C. Weng, and Y. Zhang, "VaR and CTE criteria for optimal quota-share and stop-loss reinsurance," North American Actuarial Journal, vol. 13, no. 4, pp. 459-482, 2009.

[12] K. S. Tan, C. Weng, and Y. Zhang, "Optimality of general reinsurance contracts under CTE risk measure," Insurance: Mathematics \& Economics, vol. 49, no. 2, pp. 175-187, 2011.

[13] K. C. Cheung, "Optimal reinsurance revisited-a geometric approach," ASTIN Bulletin, vol. 40, no. 1, pp. 221-239, 2010.

[14] Y. Chi and K. S. Tan, "Optimal reinsurance under VaR and CVaR risk measures: a simplified approach," ASTIN Bulletin, vol. 41, no. 2, pp. 487-509, 2011.

[15] Y. Chi and K. S. Tan, "Optimal reinsurance with general premium principles," Insurance: Mathematics and Economics, vol. 52, no. 2, pp. 180-189, 2013.

[16] R. T. Marler and J. S. Arora, "Survey of multi-objective optimization methods for engineering," Structural \& Multidisciplinary Optimization, vol. 26, no. 6, pp. 369-395, 2004.
[17] F. B. Abdelaziz, B. Aouni, and R. E. Fayedh, "Multi-objective stochastic programming for portfolio selection," European Journal of Operational Research, vol. 177, no. 3, pp. 1811-1823, 2007.

[18] G. G. Booth and W. Bessler, "Goal programming models for managing interest-rate risk," Omega, vol. 17 , no. 1, pp. 81-89, 1989.

[19] B. M. Wheeler and J. R. M. Russel, "Goal programming and agricultural planning," Journal of the Operational Research Society, vol. 28, no. 1, pp. 21-32, 1977.

[20] L. N. Killough and T. L. Souders, "A goal programming model for public accounting firms," Accounting Review, vol. 48, no. 2, pp. 268-279, 1973.

[21] C. Zopounidis, E. Galariotis, M. Doumpos, S. Sarri, and K. Andriosopoulos, "Multiple criteria decision aiding for finance: an updated bibliographic survey," European Journal of Operational Research, vol. 247, no. 2, pp. 339-348, 2015.

[22] S. Wang, "Premium calculation by transforming the layer premium density," ASTIN Bulletin, vol. 26, no. 1, pp. 71-92, 1996.

[23] E. Jouini, W. Schachermayer, and N. Touzi, "Optimal risk sharing for law invariant monetary utility functions," Mathematical Finance, vol. 18, no. 2, pp. 269-292, 2008.

[24] C. Burgert and L. Ruschendorf, "Allocation of risks and equilibrium in markets with finitely many traders," Insurance: Mathematics and Economics, vol. 42, no. 1, pp. 177-188, 2008.

[25] J. Dhaene, S. Vanduffel, M. J. Goovaerts, R. Kaas, Q. Tang, and D. Vyncke, "Risk measures and comonotonicity: a review," Stochastic Models, vol. 22, no. 4, pp. 573-606, 2006.

[26] J. Cai and W. Wei, "Optimal reinsurance with positively dependent risks," Insurance: Mathematics \& Economics, vol. 50, no. 1, pp. 57-63, 2012. 


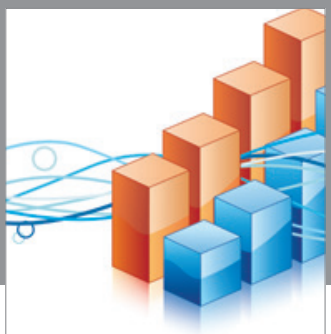

Advances in

Operations Research

vatem alat4

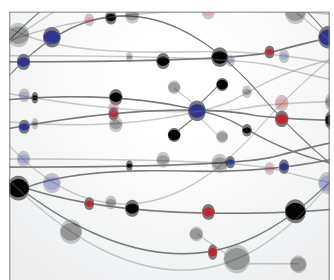

\section{The Scientific} World Journal
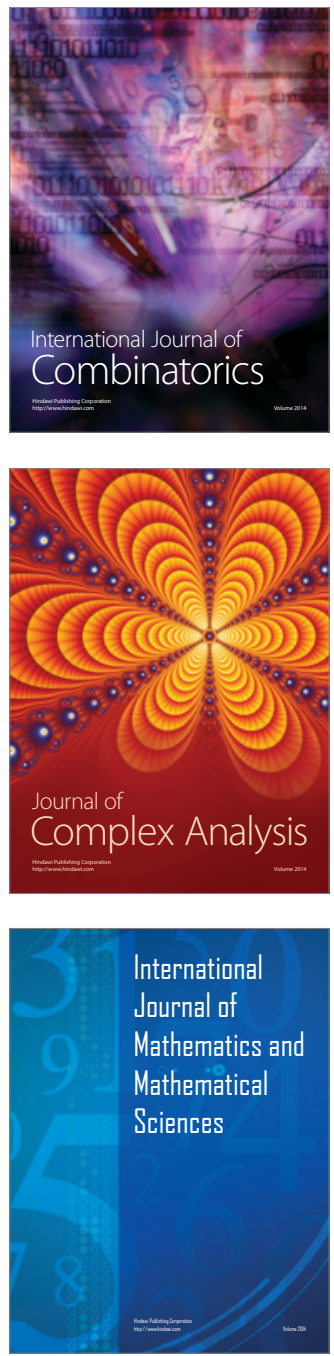
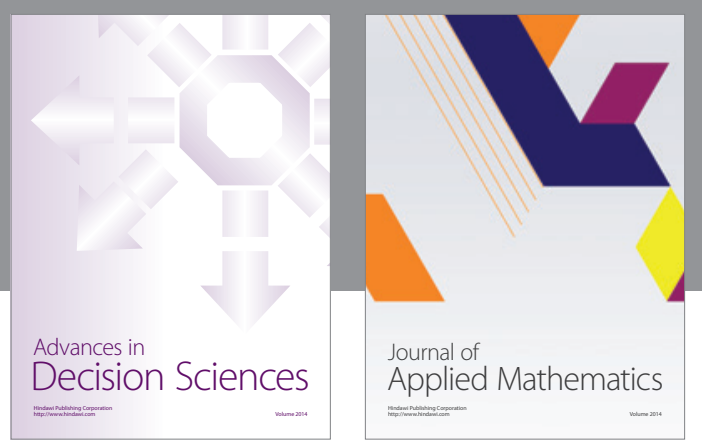

Algebra

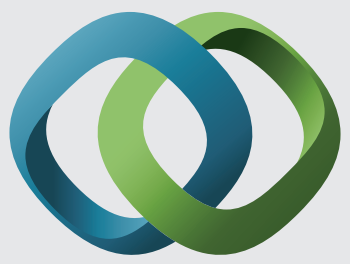

\section{Hindawi}

Submit your manuscripts at

http://www.hindawi.com
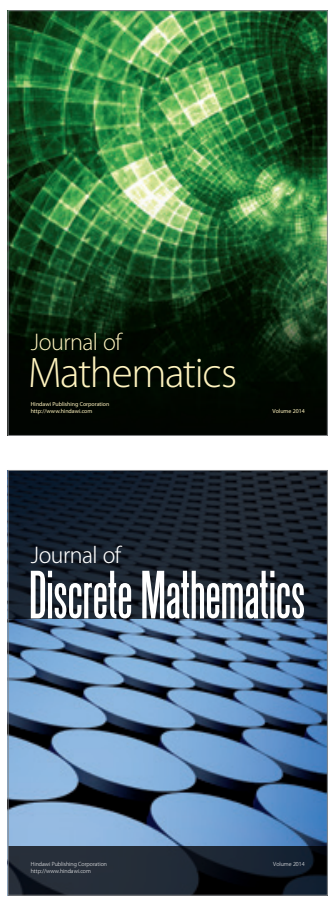

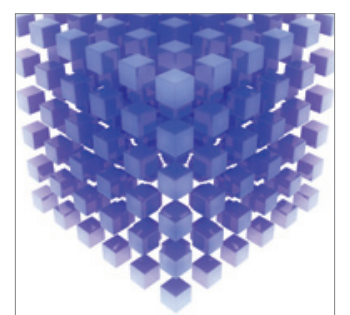

Mathematical Problems in Engineering
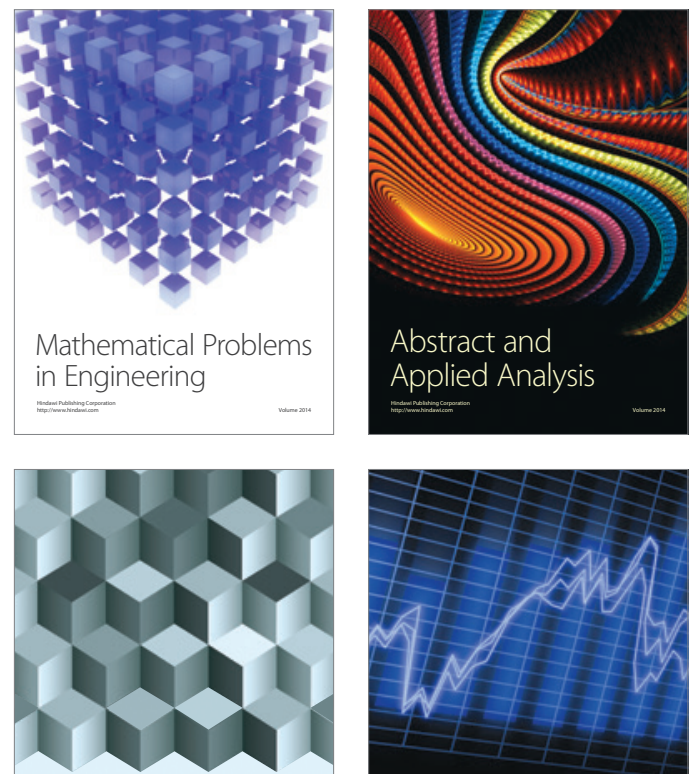

Journal of

Function Spaces

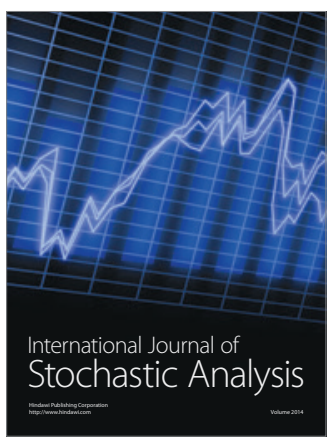

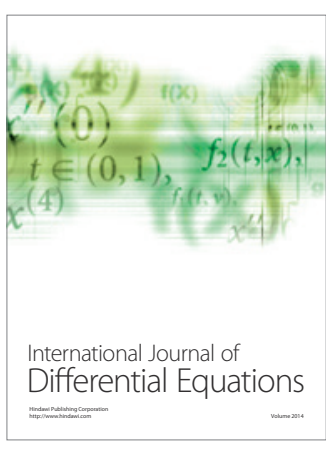
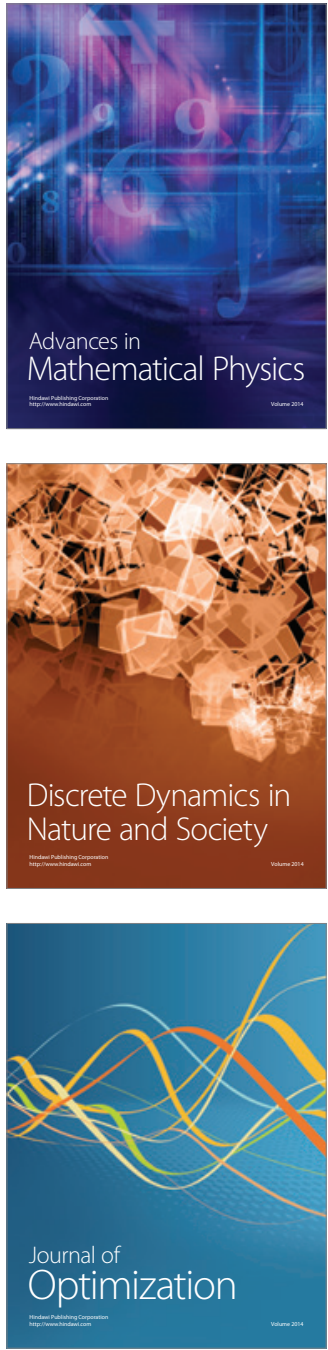